\title{
Based on UPLC-Q-TOF-MS/MS, Systematic Network Pharmacology, and Molecular Docking to Explore the Potential Mechanism of Fructus Aurantii for Major Depression Disorder
}

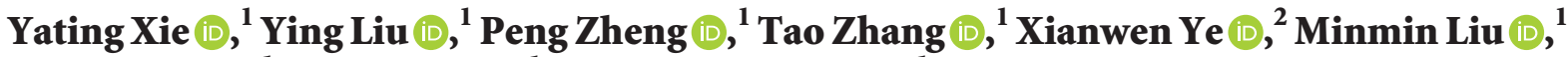

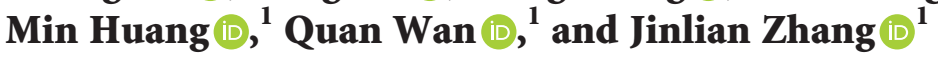 \\ ${ }^{1}$ School of Pharmacy, Jiangxi University of Chinese Medicine, Nanchang 330004, China \\ ${ }^{2}$ School of Chinese Material Medica, Beijing University of Chinese Medicine, Beijing 100102, China \\ Correspondence should be addressed to Jinlian Zhang; jxjzzjl@163.com
}

Received 22 July 2021; Accepted 20 September 2021; Published 8 October 2021

Academic Editor: Junqing Huang

Copyright (c) 2021 Yating Xie et al. This is an open access article distributed under the Creative Commons Attribution License, which permits unrestricted use, distribution, and reproduction in any medium, provided the original work is properly cited.

\begin{abstract}
Background. Major Depression Disorder (MDD) is a common mental disease that has become one of the world's major medical diseases. Currently, the Fructus Aurantii (FA) has been widely used to treat depression. However, the active substance ingredients and potential mechanisms of the shell antidepression have not yet been clarified. Method. First, we used ultraperformance liquid chromatography-quadrupole/time-of-flight tandem mass (UPLC-QTOF-MS/MS) technology to identify the chemical composition of the FA. Then, it is predicted for active ingredients, pharmaceutical disease target screening by DiscoveryStudio 2016 (DS), Metascape, and other databases, PPI network diagram, and FC core pathway. Finally, the system network pharmacology results are verified by molecular contact verification. Results. Forty-six compounds in FA were identified, and twelve active ingredients were determined. Various database information, PPI network analysis of 41 intersections, and 20 core targets including DRD2, MTOR, FASP3, and PIK3P1 were integrated. Finally, the MDD treatment is indicated by molecular docking, and the most relevant potential signal pathway is the PI3K-Akt signaling pathway.
\end{abstract}

\section{Introduction}

Major Depression Disorder (MDD) is the most common chronic mental disease nowadays, mainly manifested as depression, loss of pleasure, cognitive impairment, panic, lack of initiative, anorexia, insomnia, suicide injury, and other behaviors. Therefore, it is difficult to treat this disease due to its complexity, heterogeneity, and high recurrence [1-4]. Many records related to depression treated using traditional Chinese medicine are available, which divide MDD into six syndrome types: liver depression and spleen deficiency, liver depression and phlegm stagnation, liver depression and qi stagnation, heart and spleen deficiency, heart and kidney disharmony, and qi stagnation and blood stasis [5]. According to the latest report released by the World Health Organization (WHO), there are about 350 million people suffering from MDD in the world, with an average incidence of $4.4 \%$. It is estimated that depression will become the world's largest burden disease by $2030[6,7]$. At present, the pathogenesis of MDD has not yet been established, and the conventional western medicine uses fluoxetine hydrochloride and sertraline, which are exerting side effects, so it is difficult for them to achieve satisfactory treatment effect. In general, antidepressants cause side effects that ruin the normal daily life, so that patients have poor compliance.

Therefore, in this work, the multicomponent approach used by the traditional Chinese medicine was compared to the traditional treatment of MDD in consideration of the shortcomings of western medicine.

Fructus Aurantii (FA) is the dry immature fruit of the Citrus Aurantium L. It often used as a Chinese medicine for regulating qi and used in clinical practice and is one of the components of the most common Chinese medicine prescriptions, including Chaihu Shugan San and Weichang 
Anwan $[8,9]$. The main active ingredients of the FA include flavonoids, coumarins, volatile oils, and alkaloids, which exert an antidepressant effect [10], lower the cholesterol [11], have antitumor effect [12], promote the gastrointestinal power [13], and have other pharmacological activities. Psychological stress due to MDD can lead to gastric emptying and abnormal gastrointestinal hormone levels; FA not only has antidepressant effects, but also promotes the gastrointestinal power by regulating the hypothalamic-pituitary-adrenal axis, alleviating the discomfort caused by MDD [14]. However, the mechanism of action of FA on MDD is not yet clear. Therefore, this study explored the potential mechanism of FA on MDD using UPLC-QTOF-MS/MS, system network pharmacology, and molecular docking (Figure 1).

The FA is mainly produced in the province of Jiangxi, and it is of the highest quality. Therefore, this experiment used FA as raw material, and the chemical composition was determined by UPLC-QTOF-MS/MS. In addition, the active ingredient, disease target, and the action pathway were investigated to obtain the underlined mechanism used by FA on MDD, which was further verified by molecular docking [15-17].

\section{Materials and Methods}

2.1. Chemicals, Agents, and Materials. FA was purchased in Yicheng Town, Zhangshu City, Jiangxi Province (Jiangxi, China).

Professor Geifei, who is the head of the Chinese Medicine Resources Discipline Group, at the Jiangxi University of Traditional Chinese Medicine characterized the dry unripe fruit of Citrus aurantium L. Twelve pure compounds (purity >98\%) such as eriodictyol, 5-demethylnobiletin, naringenin, nobiletin, $3^{\prime}, 4^{\prime}, 3,5,6,7,8$-heptamethoxyflavone, auraptene, ferulic acid, umbelliferone, hesperidin, naringin, neohesperidin, and limonin were selected according to the 2015 "Chinese Pharmacopoeia" method to process FA and purchased from Sichuan Vicky Biotechnology Co. Ltd. (Sichuan, China). Methanol was purchased from Xilong Scientific Co, Ltd. (Guangdong, China). Acetonitrile (Tedia, USA), formic acid (ACS), and ethanol were purchased from the National Medicine Group Chemical Reagent Co., Ltd. (Shanghai, China). The water used in this work was ultrapure, obtained by the Milli-QB system.

\section{UPLC-QTOF-MS/MS}

3.1. Preparation of the Standard and Sample Solutions. The impurities were removed from FA, then it was washed, moisturized, and cut into thin slices, and the broken core was sifted out after drying [18]. FA crude powder $(10 \mathrm{~g})$ was precisely weighed and placed in a conical bottle with $100 \mathrm{ml}$ $70 \%$ ethanol, thoroughly mixed, socked for $0.5 \mathrm{~h}$, and boiled under reflux for $1.5 \mathrm{~h}$, and the filtrate was collected. In the above methods, $80 \mathrm{~mL} 70 \%$ ethanol and $60 \mathrm{ml} 70 \%$ ethanol were used. Three filtrates were mixed, and the filtrate was condensed and made into extract. Then, $1 \mathrm{~g}$ concrete was weighed and placed in $100 \mathrm{ml}$ methanol to dilute it. Finally, the solution was filtered through a $0.22 \mathrm{~mm}$ microporous membrane.
Twelve milligrams of each reference compound (eriodictyol; 5-demethylnobiletin; naringenin; nobiletin; $3^{\prime}, 4^{\prime}, 3$, 5, 6, 7, 8-heptamethoxyflavone; auraptene; ferulic acid; umbelliferone; hesperidin; naringin; neohesperidin; and limonin) was weighed, transferred to $10 \mathrm{ml}$ volumetric flasks, and diluted with methanol to reach the volumetric mark. The solutions were filtered through a $0.22 \mu \mathrm{m} \mathrm{mi-}$ croporous membrane to obtain the standard solutions.

\subsection{Ultraperformance Liquid Chromatography-Quadrupole-} Time-of-Flight Tandem Mass Conditions. Chemical analysis was conducted on a UPLC- (Nexera X2 LC-30A, Shimadzu Corp., Japan) hybrid triple quadrupole-time-of-flight mass spectrometer (Triple TOF ${ }^{\mathrm{TM}} 5600+$, AB Sciex, Forster City, FA, USA) connected with an electrospray ionization source (ESI). Acquity UPLC BEH C18 column $(2.1 \times 100 \mathrm{~mm} \times 1.7 \mu \mathrm{m})$ was used to perform the chromatographic separation with a flow rate of $0.3 \mathrm{ml} / \mathrm{min}$ at $40^{\circ} \mathrm{C}$. A linear gradient program with a mobile phase system including solvent A (100\% acetonitrile, v/ v) and solvent B (0.01\% formic acid in water, v/v) was performed as follows: solvent A at 5\% 20\% for 0.01 2 $\mathrm{min}, 20 \%$ $30 \%$ for $2 \sim 10 \mathrm{~min}, 30 \sim 55 \%$ for $10 \sim 25 \mathrm{~min}, 55 \% \sim 100 \%$ for 25 30 $\mathrm{min}, 100 \%$ A for 30 32 $\mathrm{min}$, and $100 \% \sim 5 \%$ for $0.5 \mathrm{~min}$, with isocratic elution performed at $5 \%$ for $2.5 \mathrm{~min}$.

The instrumental setting of Q-TOF-MS/MS was the following: ion source gas 1 (GSI) and gas 2 (GS2) were both set to $60 \mathrm{kPa}$, curtain gas (CUR) was set to $35 \mathrm{kPa}$, ion spray voltage floating (ISVF) was set to $5500 \mathrm{~V}$, ion source temperature (TEM) was set at $500^{\circ} \mathrm{C}$, collision energy (CE) was set at $45 \mathrm{eV}$, collision energy spread (CES) was set at $45 \pm 10 \mathrm{eV}$, the declustering potential (DP) was set at $100 \mathrm{~V}$, and nitrogen was used as a nebulizer and auxiliary gas. Samples were analyzed in positive ionization modes with a sFAnnin mass-to-charge $(\mathrm{m} / \mathrm{z})$ range from 50 to 1,000 . Data were collected in information-dependent acquisition (IDA) mode and analyzed by PeakView ${ }^{\circledR} 1.2$ software (AB Sciex).

3.3. Ingredients Identification Analysis. The chemical composition of FA was obtained from existing databases and documents, including TCMSP, SCHINDER, TCMIP, China Knowledge Network, and Geen Medical. The data were sorted out and the FA composition database was established. (+) MS data was imported into PeakView ${ }^{\circledR} 1.2$, "XIC Manager" was used to analyze the $70 \%$ ethanol extract of FA, and each chemical composition in the retention time and its corresponding primary and secondary mass spectrum data were obtained. It combined the base peak chart with the reference substance and compared it with the data reported in the relevant references, and the chemical composition was confirmed.

\subsection{Identification of the Related Targets of FA Components.} In this study, the chemical compounds were imported into Discovery Studio 2016 (DS), through "ADMET Descriptors" to screen the active ingredients. The related targets of FA components were obtained from SwissTargetPrediction (https://www.swisstargetprediction.ch/) and Pharmmapper (https://www.lilab-ecust.cn/pharmmapper/). 


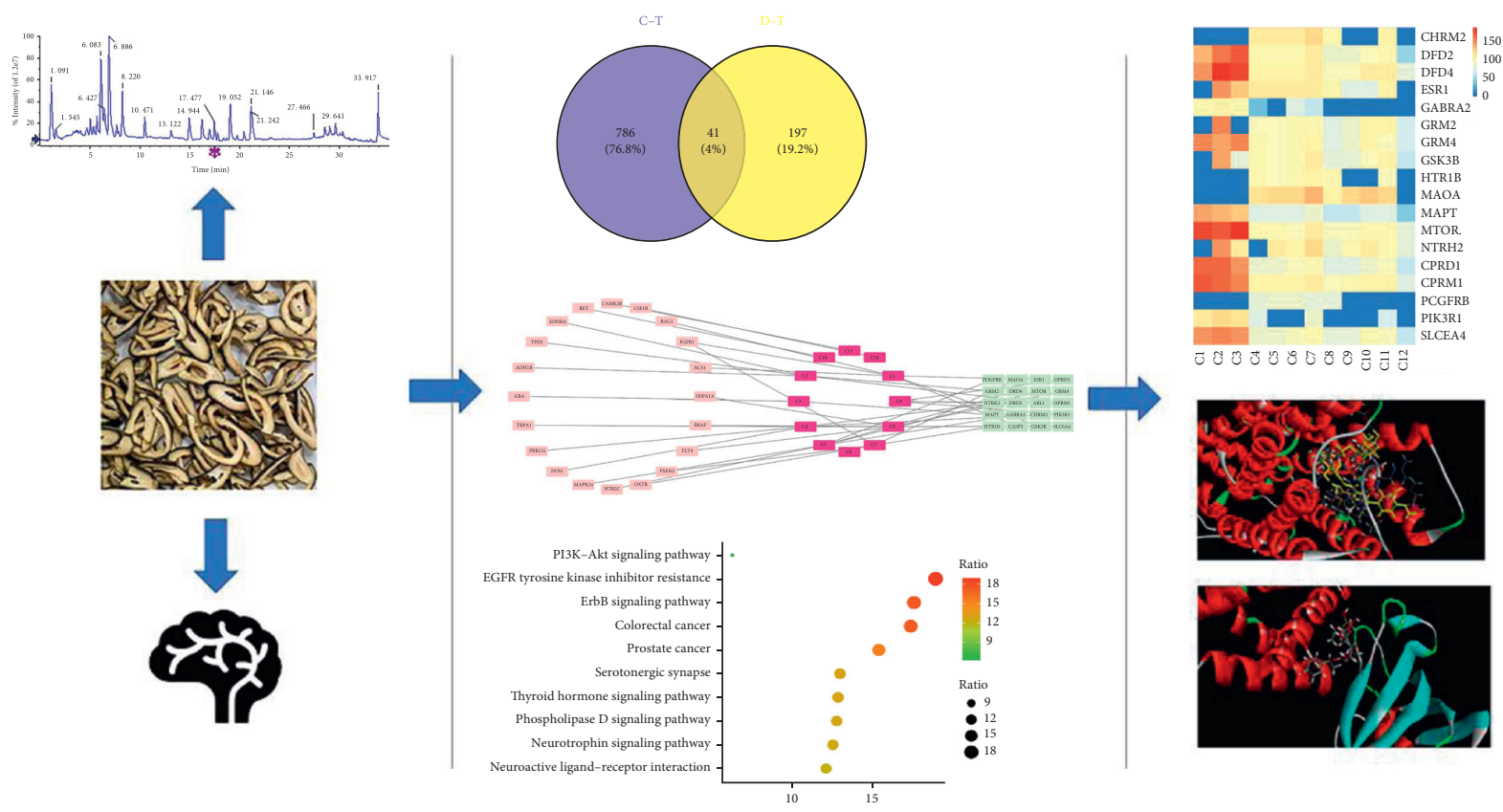

FIGURE 1: Based on systems pharmacology strategy to study the mechanism of FA in treatment of MDD.

3.5. MDD-Associated Targets Collection. The keyword "depression" was used in the Genecards database (https://www. geneFArds.org/) and Online Mendelian Inheritance in Man (OMIM, https://omim.org/) to identify disease targets associated with MDD.

3.6. Construction of the Protein-Protein Interaction Network. VENNY2.1 software (https://bioinfogp.cnb.csic.es/tools/ venny/index.html) was used to obtain the overlapping targets between FA-related targets and MDD-related targets. Overlapping targets were imported into the Retrieval of Interacting Genes/Proteins (STRING) 11.0 (https://www. string-db.org/), and the interaction results were saved. The interaction resulting file was added to Cytoscape v3.9.0 software, and the Protein-Protein Interaction (PPI) network was obtained.

3.7. Go Function and Genomes (KEGG) Pathway Enrichment Analysis. Metascape is a powerful tool for the analysis of gene function annotation. It applies the bioinformatics analysis method to a large number of genes or proteins, and it uses more than 40 independent databases to annotate genes or proteins, for the enrichment analysis and the construction of the PPI network. WebGestalt was used to perform the enrichment analysis. The software can meet user requirements from different areas, by pathway figure and hierarchical network visualization. Since its release in 2005, it has gradually become one of the most popular software types in the field of biology [19].

Overlapping targets were imported into Metascape (https://www.metascape.org/), "Homo sapiens" was selected as "Organism of interest," and "Pathway" and "KEGG" were selected as "Functional Database." Finally, "genome" was selected as the "Select reference set," and the KEGG pathway analysis was performed.

3.8. I-D-G Network Construction. Related files were established as "core ingredients-core targets," "core ingredients-Secondary targets," "disease-core targets," and "disease-Secondary targets." Then, the files were imported into Cytoscape v3.9.0 to build a "ingredients-disease-gene symbols" network.

3.9. Computational Validation of I-T Interactions. The computational software was used to simulate the interaction between active compounds and core targets and to explore the binding ability and binding mode between compounds and targets. Therefore, 12 drug components were selected for the molecular docking with 14 core targets. The PDB format of the target protein was downloaded from the structural bioinformatics protein database (PDB, https://www.rcsb. org/). The protein structure was used to simulate dehydration, hydrogenation, and removal of proteins using DiscoveryStudio2016 (DS) software, and then molecular docking was performed.

\section{Results}

4.1. Identification of the Chemical Constituents in FA by UPLC-QTOF-MS/MS. UPLC-QTOF-MS/MS is an analytical technique widely used in drug research, which can perform chromatography and identify fragment ions and cleavage patterns. Figure 2 represents a (+) ESI-MS mass total ion chromatogram of the FA extract (TIC). A total of 46 compounds were identified using PeakView ${ }^{\circledR} 1.2$ software and literature comparison, including 33 flavonoids, 10 coumarins, 3 limonoids, and other compounds. Twelve 


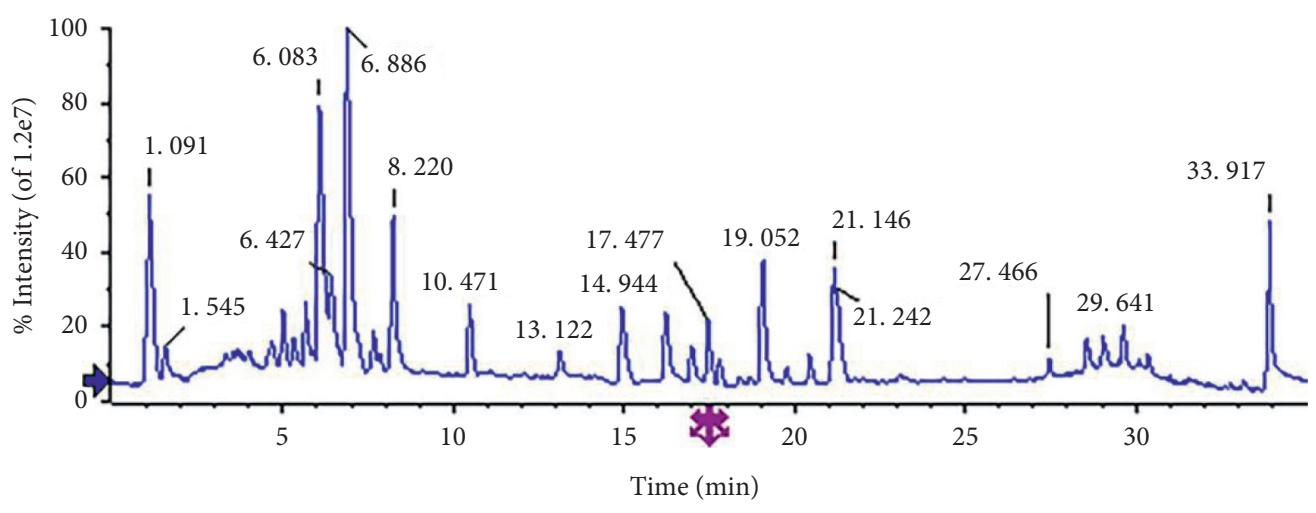

Figure 2: The (+) ESI-MS mass total ion chromatogram (TICs) of the FA by UPLC/Q-TOF-MS/MS.

ingredients (eriodictyol; 5-demethylnobiletin; naringenin; nobiletin; $3^{\prime}, 4^{\prime}, 3,5,6,7,8$-heptamethoxyflavone; auraptene; ferulic acid; umbelliferone, hesperidin; naringin; neohesperidin; limonin) were confirmed by the comparison with the reference substance (Table 1 ).

4.2. Screening of the Active Components in FA. The bloodbrain barrier (BBB) is an important structure in the human body to maintain a stable brain environment; thus, lipophilic and water-soluble drugs do not cross easily the BBB, limiting the effect of $95 \%$ of drugs on the central nervous system [25]. Depression is a neurological disorder occurring in the central nervous system; thus, the relationship between the ability of antidepressants and the permeability of the BBB is of utmost importance to obtain an effective result.

Forty-six compounds were screened by DS software, and the screening standard used was "ADMET_BBB $\geq-0.3$ " [26]. Seven active compounds of FA were obtained, such as isomerancin, meranzin, prangenin, bergapten, 5, 6, 7, 4'tetramethoxyflavone, $3^{\prime}, 4^{\prime}, 7,8$-tetramethoxyflavone, and Scutellarein. Scutellarein has a good permeability in the BBB model in vitro, has the ability to improve BBB dysfunction, and treats stroke, Alzheimer's disease, and other central nervous system diseases $[27,28]$. Bergapten has extremely high bioavailability, can cross the BBB, protect nerves, and treat brain diseases, and it is a potential candidate as antidepressant drug [29]. However, some compounds not meeting the screening criteria were also chosen in order to comprehensively evaluate MDD treatment. For example, naringin and neohesperidin are the main components of FA according to the 2020 "China Pharmacopoeia." In addition, according to the literature, meranzin hydrate and nobiletin can reduce ROS, MDA, IL-6, and TNF- $\alpha$ levels in MDD rats; these compounds can cause anti-inflammatory and antioxidative stress [30]. Naringin can improve the hippocampus mTOR and P70S6K phosphorylation level [31]. Hesperidin can improve CUMS rat behavior by regulating the function of the hypothalamic-pituitary-adrenal (HPA) axis [32]. Although these compounds do not satisfy the screening criteria of crossing the BBB, some studies showed their biological activity; thus, these 5 compounds were also used as candidate active components. Finally, 12 compounds were chosen as active ingredients (Table 2).
4.3. Acquisition of the Related Targets of FA Components. All the targets of the compounds in FA were collected from the SwissTargetPrediction software and Pharmmapper software. A total of 827 FA-related targets were obtained after the removal of the repeated targets.

4.4. Identification of the MDD-Associated Targets. The screening standard used was "Relevance score $>10$ " [33], which allowed obtaining 71 and 189 MDD-related targets by Genecard database and OMIM database, respectively. A total of 238 known MDD-related targets were collected after the removal of the redundant information.

4.5. PPI Network Analysis. The disease targets and composition targets were combined and imported into Venny2.1 software, and 41 overlapping targets were obtained by the Venny diagram (Figure 3(a)) and PPI network (Figure 3(c)). The PPI network diagram was visualized by the Cytoscape software for a further analysis (Figure 3(b)). The results showed that NCS1 did not intersect with other targets. "Degree" could allow the evaluation of the effect to each target to a certain extent; thus, the median value of "degree" was used as a screening condition [34], and degree was ranked as a core target (Figure 3(d)). For example, DRD2, FASP3, and PIK3P1 could be important research targets for the treatment of MDD with FA.

4.6. Analysis of the Go and KEGG Pathway Enrichment. WebGestalt and Metascape were used to perform Go function and KEGG pathway enrichment analysis on 20 core targets. The results showed the involvement of 12 Biological Processes (BP), 16 Cellular Components (CC), and 13 Molecular Functions (MF). BP was related to cell communication, response to stimulus, and biological regulation, CC was related to membrane, cell projection, and endomembrane system, and MF was related to protein binding, molecular transducer activity, and ion binding (Figure 4(a)).

There is a close relationship between the core target and the signaling pathway. The screening results with "value of enrichment ratio" resulted in 10 pathways, including EGFR tyrosine kinase inhibitor resistance (hsa04080), ErbB signaling pathway (hsa04072), thyroid hormone signaling pathway 
TABLE 1: Identification of chemical constituents of FA immaturus ethanol extract.

\begin{tabular}{|c|c|c|c|c|c|c|c|}
\hline No & $\begin{array}{l}\text { Molecular } \\
\text { formula }\end{array}$ & $\begin{array}{l}\text { Extraction mass } \\
\text { (Da) }\end{array}$ & $\begin{array}{l}\text { Error } \\
(\mathrm{ppm})\end{array}$ & $\begin{array}{c}\mathrm{tR} / \\
(\mathrm{min})\end{array}$ & Identity & Classification & Ref. \\
\hline 1 & $\mathrm{C}_{27} \mathrm{H}_{30} \mathrm{O}_{15}$ & 511.1658 & 2.8 & 3.73 & Lonicerin & Flavonoid & [20] \\
\hline 2 & $\mathrm{C}_{15} \mathrm{H}_{12} \mathrm{O}_{6}$ & 289.0707 & 0.2 & 4.98 & Eriodictyol & Coumarin & {$[21]$} \\
\hline 3 & $\mathrm{C}_{22} \mathrm{H}_{24} \mathrm{O}_{9}$ & 433.1493 & 1.6 & 20.45 & $3^{\prime}, 4^{\prime}, 3,5,6,7,8$-Heptamethoxyflavone & Flavonoid & {$[22]$} \\
\hline 4 & $\mathrm{C}_{27} \mathrm{H}_{32} \mathrm{O}_{15}$ & 597.1814 & 2.3 & 4.99 & Neoeriocitrin & Flavonoid & [20] \\
\hline 5 & $\mathrm{C}_{27} \mathrm{H}_{32} \mathrm{O}_{15}$ & 597.1814 & 2.3 & 4.7 & Eriocitrin & Flavonoid & [20] \\
\hline 6 & $\mathrm{C}_{10} \mathrm{H}_{10} \mathrm{O}_{4}$ & 195.0652 & -2.8 & 5.37 & Ferulic acid & $\begin{array}{l}\text { Total phenolic } \\
\text { acids }\end{array}$ & {$[20]$} \\
\hline 7 & $\mathrm{C}_{16} \mathrm{H}_{14} 0_{6}$ & 303.0863 & 0.6 & 6.88 & Hesperetin & Flavonoid & [21] \\
\hline 8 & $\mathrm{C}_{27} \mathrm{H}_{32} \mathrm{O}_{14}$ & 581.1865 & 2.4 & 5.67 & Narirutin & Flavonoid & {$[21]$} \\
\hline 9 & $\mathrm{C}_{15} \mathrm{H}_{12} \mathrm{O}_{5}$ & 273.0758 & -1 & 12.04 & Naringenin & Flavonoid & {$[21]$} \\
\hline 10 & $\mathrm{C}_{21} \mathrm{H}_{22} \mathrm{O}_{10}$ & 435.1286 & 1.2 & 6.07 & Naringenin-7-O-glucoside & Flavonoid & {$[20]$} \\
\hline 11 & $\mathrm{C}_{22} \mathrm{H}_{24} \mathrm{O}_{11}$ & 465.1391 & 0.8 & 6.87 & Hesperetin-7-O-glucoside & Flavonoid & [21] \\
\hline 12 & $\mathrm{C}_{28} \mathrm{H}_{34} \mathrm{O}_{15}$ & 611.1971 & 1.6 & 6.42 & Hesperidin & Flavonoid & [20] \\
\hline 13 & $\mathrm{C}_{27} \mathrm{H}_{32} \mathrm{O}_{14}$ & 581.1865 & 2.4 & 6.06 & Naringin & Flavonoid & {$[23]$} \\
\hline 14 & $\mathrm{C}_{28} \mathrm{H}_{34} \mathrm{O}_{15}$ & 611.1971 & 1.6 & 6.87 & Neohesperidin & Flavonoid & {$[23]$} \\
\hline 15 & $\mathrm{C}_{22} \mathrm{H}_{24} \mathrm{O}_{11}$ & 465.1391 & 0.8 & 7.24 & Eriodictiol-7-O-glucoside & Flavonoid & {$[21]$} \\
\hline 16 & $\mathrm{C}_{15} \mathrm{H}_{16} \mathrm{O}_{4}$ & 261.1121 & -0.9 & 16.22 & Isomerancin & Coumarin & [20] \\
\hline 17 & $\mathrm{C}_{15} \mathrm{H}_{16} \mathrm{O}_{4}$ & 261.1121 & -0.9 & 8.22 & Meranzin & Coumarin & {$[20]$} \\
\hline 18 & $\mathrm{C}_{15} \mathrm{H}_{18} \mathrm{O}_{5}$ & 279.1227 & -0.1 & 8.23 & Meranzin hydrate & Coumarin & {$[20]$} \\
\hline 19 & $\mathrm{C}_{11} \mathrm{H}_{6} \mathrm{O}_{4}$ & 203.0339 & -2.7 & 9.36 & Xanthotoxol & Coumarin & {$[20]$} \\
\hline 20 & $\mathrm{C}_{26} \mathrm{H}_{27} \mathrm{O}_{14}$ & 564.1474 & 2.6 & 9.86 & Isonaringin & Flavonoid & {$[20]$} \\
\hline 21 & $\mathrm{C}_{16} \mathrm{H}_{14} \mathrm{O}_{5}$ & 287.0914 & -0.8 & 10.45 & Prangenin & Coumarin & {$[21]$} \\
\hline 22 & $\mathrm{C}_{16} \mathrm{H}_{14} \mathrm{O}_{5}$ & 287.0914 & -0.8 & 10.47 & Isosakuranetin & Flavonoid & {$[21]$} \\
\hline 23 & $\mathrm{C}_{28} \mathrm{H}_{34} \mathrm{O}_{14}$ & 595.2021 & 1.7 & 6.88 & $\begin{array}{l}\text { Poncirin (isosakuranetin-7-O- } \\
\text { neohesperidoside) }\end{array}$ & Flavonoid & [21] \\
\hline 24 & $\mathrm{C}_{28} \mathrm{H}_{34} \mathrm{O}_{14}$ & 595.2021 & 1.7 & 8.22 & Neoponcirin & Flavonoid & {$[21]$} \\
\hline 25 & $\mathrm{C}_{28} \mathrm{H}_{34} \mathrm{O}_{14}$ & 595.2021 & 1.7 & 9.85 & Isosakuranetin-7-O-rutinoside & Flavonoid & {$[21]$} \\
\hline 26 & $\mathrm{C}_{28} \mathrm{H}_{34} \mathrm{O}_{14}$ & 595.2021 & 1.7 & 5.89 & Poncirin & Flavonoid & {$[20]$} \\
\hline 27 & $\mathrm{C}_{18} \mathrm{H}_{16} \mathrm{O}_{6}$ & 329.102 & 0.3 & 11.53 & $4^{\prime}$-Hydroxy-5,6,7-trimethoxyflavone & Flavonoid & [22] \\
\hline 28 & $\mathrm{C}_{12} \mathrm{H}_{8} \mathrm{O}_{4}$ & 217.0495 & -2.3 & 14.77 & Bergapten & Coumarin & [20] \\
\hline 29 & $\mathrm{C}_{19} \mathrm{H}_{24} \mathrm{O}_{5}$ & 333.1697 & 0.5 & 14.93 & Marmin & Coumarin & {$[20]$} \\
\hline 30 & $\mathrm{C}_{19} \mathrm{H}_{18} \mathrm{O}_{6}$ & 343.1176 & -0.5 & 16.98 & $5,7,8,4^{\prime}$-Tetramethoxyflavone & Flavonoid & {$[22]$} \\
\hline 31 & $\mathrm{C}_{19} \mathrm{H}_{18} \mathrm{O}_{6}$ & 343.1176 & -0.5 & 16.99 & 5,6,7,4'-Tetramethoxyflavone & Flavonoid & {$[22]$} \\
\hline 32 & $\mathrm{C}_{19} \mathrm{H}_{18} \mathrm{O}_{6}$ & 343.1176 & -0.5 & 19.08 & $3^{\prime}, 4^{\prime}, 7,8$-Tetramethoxyflavone & Flavonoid & {$[22]$} \\
\hline 33 & $\mathrm{C}_{19} \mathrm{H}_{18} \mathrm{O}_{6}$ & 343.1176 & -0.5 & 11.08 & Scutellarein & Flavonoid & {$[21]$} \\
\hline 34 & $\mathrm{C}_{21} \mathrm{H}_{22} \mathrm{O}_{8}$ & 403.1387 & 0.6 & 19.04 & $3,5,7,8,3^{\prime}, 4^{\prime}$-Hexamethoxyflavone & Flavonoid & {$[22]$} \\
\hline 35 & $\mathrm{C}_{21} \mathrm{H}_{22} \mathrm{O}_{8}$ & 403.1387 & 0.6 & 19.05 & Nobiletin & Flavonoid & {$[20]$} \\
\hline 36 & $\mathrm{C}_{28} \mathrm{H}_{34} \mathrm{O}_{9}$ & 515.2276 & 1 & 19.75 & Nomilin & Limonoid & {$[22]$} \\
\hline 37 & $\mathrm{C}_{20} \mathrm{H}_{20} \mathrm{O}_{7}$ & 373.1282 & 0.7 & 15.04 & $5,7,8,3^{\prime}, 4^{\prime}$-Pentamethoxyflavone & Flavonoid & {$[22]$} \\
\hline 38 & $\mathrm{C}_{20} \mathrm{H}_{20} \mathrm{O}_{7}$ & 373.1282 & 0.7 & 16.72 & Auranetin & Flavonoid & {$[21]$} \\
\hline 39 & $\mathrm{C}_{20} \mathrm{H}_{20} \mathrm{O}_{7}$ & 373.1282 & 0.7 & 21.15 & Tangeretin & Flavonoid & {$[23]$} \\
\hline 40 & $\mathrm{C}_{20} \mathrm{H}_{20} \mathrm{O}_{7}$ & 373.1282 & 0.7 & 16.97 & Sinensetin & Flavonoid & {$[20]$} \\
\hline 41 & $\mathrm{C}_{20} \mathrm{H}_{20} \mathrm{O}_{7}$ & 373.1282 & 0.7 & 16.72 & Isosinensetin & Flavonoid & {$[21]$} \\
\hline 42 & $\mathrm{C}_{21} \mathrm{H}_{22} \mathrm{O}_{9}$ & 419.1337 & 1.1 & 21.27 & Natsudaidain & Flavonoid & {$[20]$} \\
\hline 43 & $\mathrm{C}_{20} \mathrm{H}_{20} \mathrm{O}_{8}$ & 389.1231 & 0.2 & {$[23] .07$} & 5-Demethylnobiletin & Flavonoid & {$[22]$} \\
\hline 44 & $\mathrm{C}_{9} \mathrm{H}_{6} \mathrm{O}_{3}$ & 163.039 & -4.1 & 5.33 & Umbelliferone & Coumarin & {$[20]$} \\
\hline 45 & $\mathrm{C}_{19} \mathrm{H}_{22} \mathrm{O}_{3}$ & 299.1642 & -0.3 & 29.63 & Auraptene & Coumarin & {$[24]$} \\
\hline 46 & $\mathrm{C}_{26} \mathrm{H}_{30} \mathrm{O}_{8}$ & 471.2013 & -4 & 17.47 & Limonin & Limonoid & {$[22]$} \\
\hline
\end{tabular}

(hsa04919), PI3K-Akt signaling pathway (hsa04151), and neuroactive ligand-receptor interaction (hsa04722). Some antidepressants exert a therapeutic effect through the ErbB signaling pathway and often induce the downregulation of NRG1/ErbB4; thus, our hypothesis was that antidepressants could play a role through this channel [35]. Therefore, the above pathways were obtained by screening, providing a direction in the research of a new mechanism of action of FA.
4.7. I-D-G Network Analysis. Cytoscape v3.9.0 software was used to build an "ingredients-disease-gene symbols" network, as shown in Figure 5, in which the pink node represented the common targets, the twelve magenta nodes represented the core ingredients of FA, and the 20 green nodes represented the core gene symbols between FA and MDD. This result revealed that FA could play a role in the treatment of MDD through multiple targets. 
TABLE 2: Absorption parameters of 12 FA components.

\begin{tabular}{lccc}
\hline No & Component & Molecular formula & BBB \\
\hline $\mathrm{C} 1$ & Hesperidin & $\mathrm{C}_{28} \mathrm{H}_{34} \mathrm{O}_{15}$ & $\mathrm{ND}$ \\
$\mathrm{C} 2$ & Naringin & $\mathrm{C}_{27} \mathrm{H}_{32} \mathrm{O}_{14}$ & $\mathrm{ND}$ \\
$\mathrm{C} 3$ & Neohesperidin & $\mathrm{C}_{28} \mathrm{H}_{34} \mathrm{O}_{15}$ & $\mathrm{ND}$ \\
$\mathrm{C} 4$ & Isomerancin & $\mathrm{C}_{15} \mathrm{H}_{16} \mathrm{O}_{4}$ & -0.126 \\
$\mathrm{C} 5$ & Meranzin & $\mathrm{C}_{15} \mathrm{H}_{16} \mathrm{O}_{4}$ & -0.098 \\
$\mathrm{C} 6$ & Meranzin hydrate & $\mathrm{C}_{15} \mathrm{H}_{18} \mathrm{O}_{5}$ & -0.852 \\
$\mathrm{C} 7$ & Prangenin & $\mathrm{C}_{16} \mathrm{H}_{14} \mathrm{O}_{5}$ & -0.282 \\
$\mathrm{C} 8$ & Bergapten & $\mathrm{C}_{12} \mathrm{H}_{8} \mathrm{O}_{4}$ & -0.233 \\
$\mathrm{C} 9$ & $5,6,7,4^{\prime}$-Tetramethoxyflavone & $\mathrm{C}_{19} \mathrm{H}_{18} \mathrm{O}_{6}$ & -0.185 \\
$\mathrm{C} 10$ & $3^{\prime}, 4^{\prime}, 7,8$-Tetramethoxyflavone & $\mathrm{C}_{19} \mathrm{H}_{18} \mathrm{O}_{6}$ & -0.185 \\
$\mathrm{C} 11$ & Scutellarein & $\mathrm{C}_{19} \mathrm{H}_{18} \mathrm{O}_{6}$ & -0.185 \\
$\mathrm{C} 12$ & Nobiletin & $\mathrm{C}_{21} \mathrm{H}_{22} \mathrm{O}_{8}$ & -0.478 \\
\hline
\end{tabular}

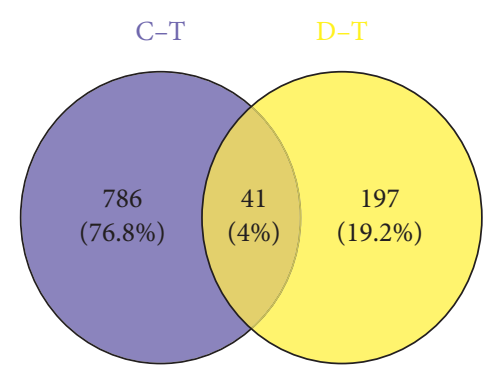

(a)

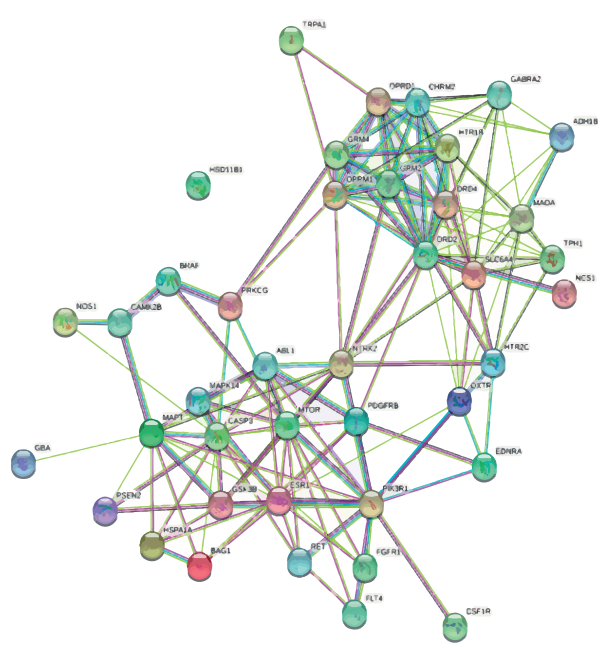

(c)

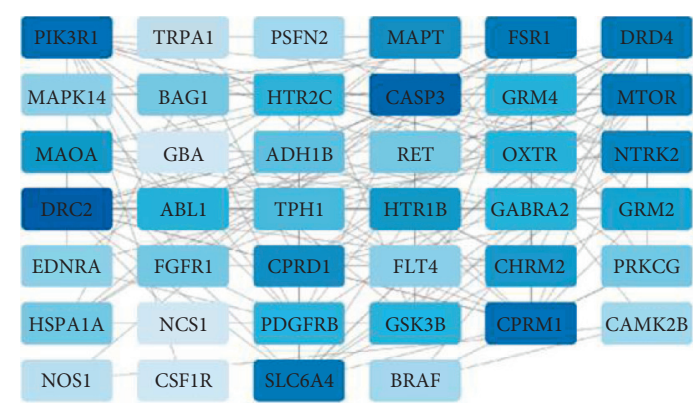

(b)

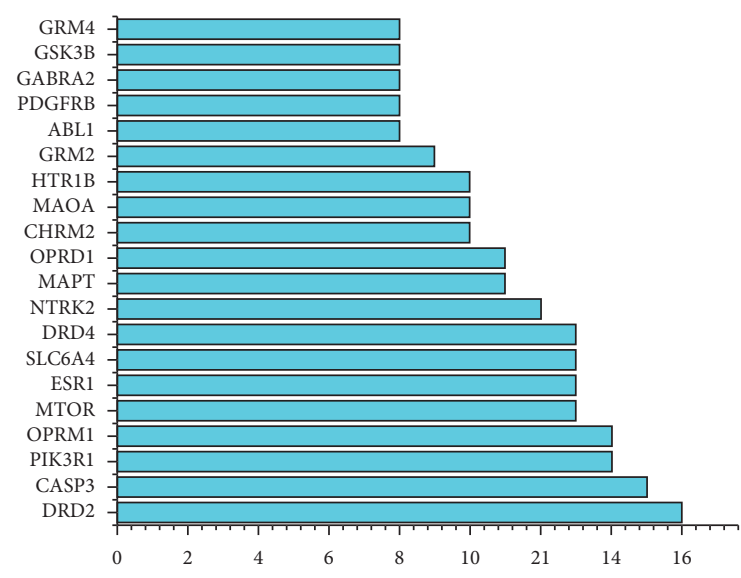

(d)

Figure 3: (a) Venn diagram of related targets of Aurantii Fructus (FA) and Major Depressive Disorder (MDD). (b) PPI network of overlapping targets between drug and disease. The more the color, the higher the value. (c) PPI network. (d) Bar plot of the 20 core targets. The $x$-axis represents the degree. The $y$-axis represents the target protein.

Cytoscape software analysis revealed that the values of C4 (isomerancin, degree $=7$ ), C1 (hesperidin, degree $=6$ ), C5 (meranzin, degree $=6$ ), C2 (naringin, degree $=5$ ), and C7 (prangenin, degree $=5$ ) were at the top of the list, meaning that these compounds could be the core components of FA effective in the treatment of MDD.

4.8. Computational Validation of I-T Interactions. The binding ability of the receptor to the ligand is closely related to the molecular docking score. The higher the score, the stronger the binding ability of the receptor. The docking scoring results showed that most of the receptors had good binding ability to the ligands except ABL1 and FASP3 genes, which had low docking scores (Table 3 ). Therefore, the two gene symbols ABL1 and FASP3 were removed during the building of the heat map. Figure 6(a) shows that the better the color, the higher the docking score. Our results revealed that neohesperidin, naringin, and hesperidin have a high binding ability with DRD4 and mTOR. 

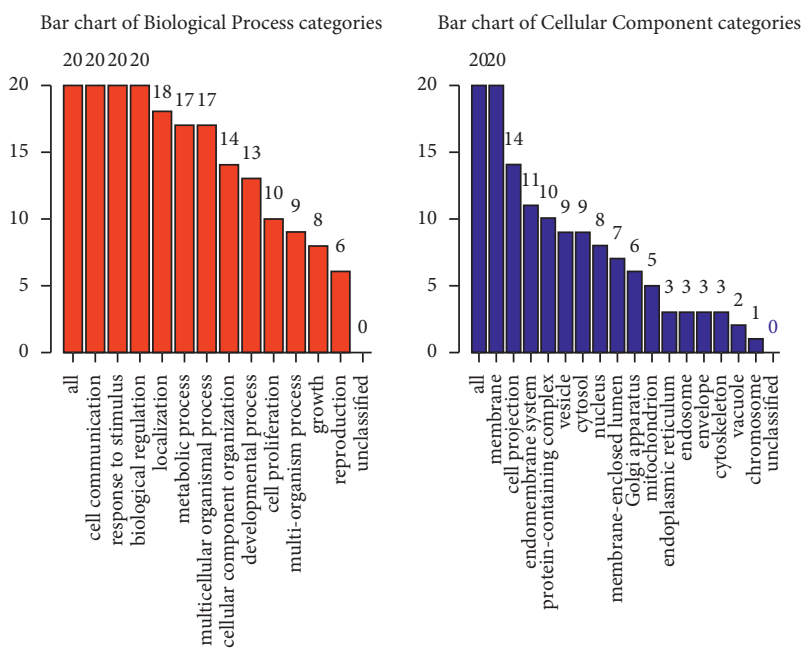

Bar chart of Molecular Function categories

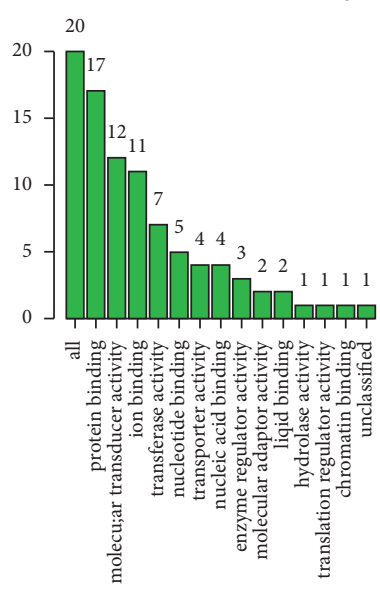

(a)

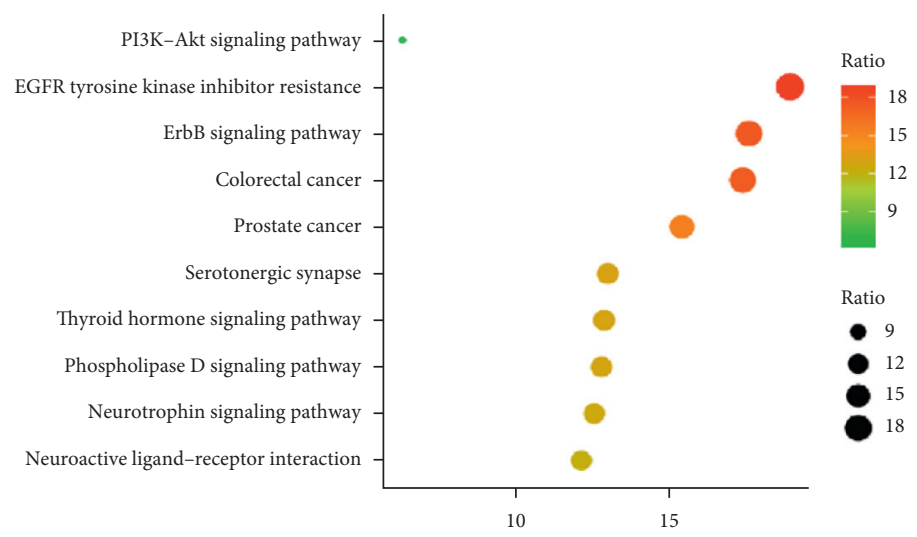

(b)

FIGURE 4: (a) Bar plot of the GO function enrichment of core targets. Red represents the Biological Process (BP). Blue represents the Cellular Component (CC). Green represents the Molecular Function (MF). (b) Bubble chart of KEGG enrichment of core targets. The $x$-axis represents the ratio enrichment. The $y$-axis represents the pathway.

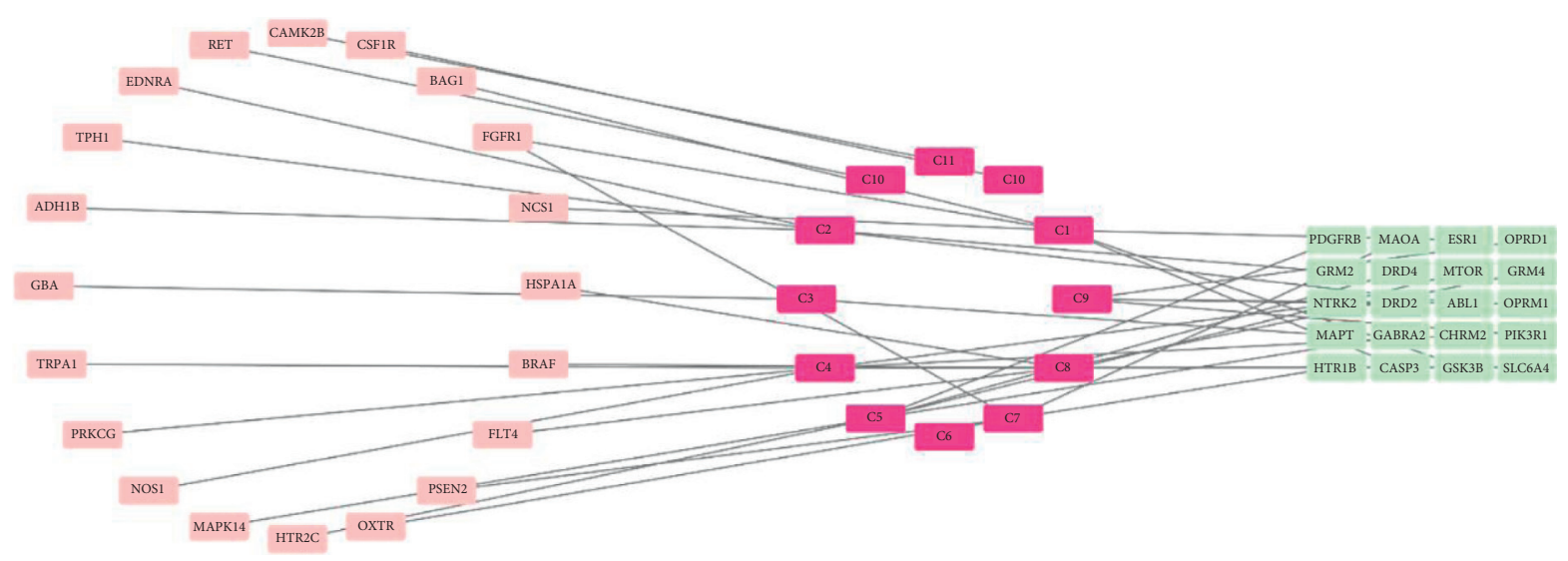

Figure 5: The I-D-G network.

Based on these data, we found that the interaction between each component and the core target is the basis of biological activity. Therefore, FA in the treatment of MDD is a complex process among a series of multitargets and multicomponents.

\section{Discussion}

MDD is a common mental disease that has become one of the world's major diseases, resulting in the change of the quality of life and work ability. The number of patients 
TABLE 3: DRD docking with the component score.

\begin{tabular}{lcr}
\hline No & Component & Score \\
\hline C2 & Naringin & 186.427 \\
C3 178.642 \\
C1 & Neohesperidin & 144.394 \\
C11 & Hesperidin & 111.055 \\
C10 & Scutellarein & 109.533 \\
C7 & $3^{\prime}, 4^{\prime}, 7$, 8-Tetramethoxyflavone & 107.563 \\
C4 & Prangenin & 103.292 \\
C5 & Isomerancin & 100.242 \\
C6 & Meranzin & 100.109 \\
C9 & Meranzin hydrate \\
C8 & $5,6,7,4^{\prime}$-Tetramethoxyflavone & 91.912 \\
C12 & Bergapten & 87.6184 \\
& Nobiletin & 77.2802 \\
\hline
\end{tabular}

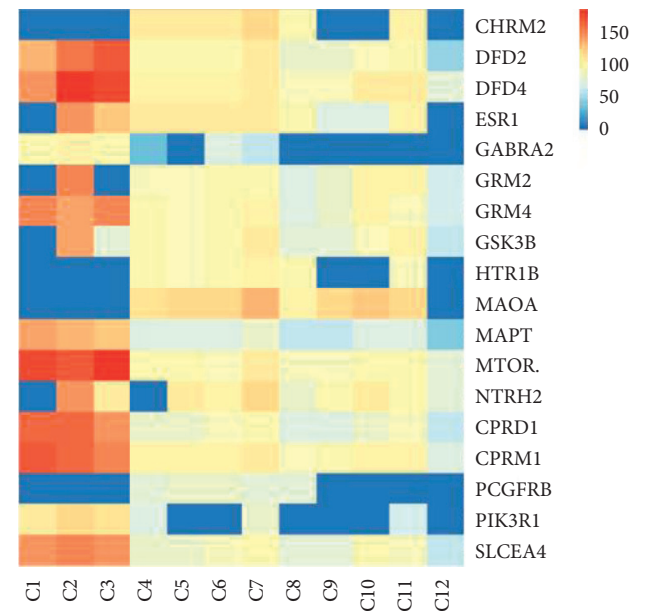

(a)
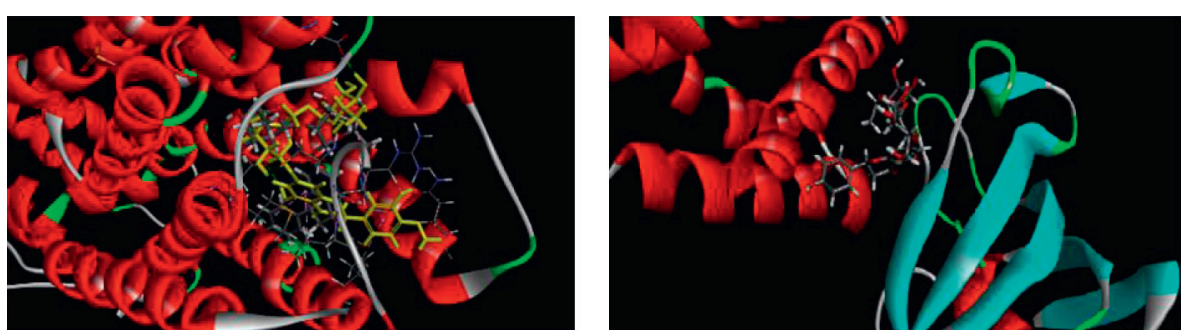

(b)

Figure 6: (a) Heatmap of I-G docking scores. (b) 3D diagram of naringin-DRD4 and naringin-mTOR.

increases year by year. At present, most of the patients suffering from clinical depression are treated by the western medicine, which commonly use selective serotonin reuptake inhibitors (fluoxetine and paroxetine) and serotonin and norepinephrine reuptake inhibitors (venlafaxine and duloxetine). However, many problems are associated with the use of the western medicine, such as high recurrence rate, poor tolerance, and adverse reactions. The treatment using traditional Chinese medicine is gradual, and the existing research results show that the curative effect of the Chinese medicine does not differ significantly from that of the western medicine. Nevertheless, the Chinese medicine has a faster effect, less adverse reactions, and more benefits. Therefore, the research on MDD of traditional Chinese medicine has been more intense and fruitful in recent years.

FA is one of the commonly used medicinal compounds, which is widely used to cure gastrointestinal diseases. Previous studies showed that MDD often leads to gastrointestinal dysfunction, and the FA extract can regulate the HPA axis [36] and neuroprotection [37], regulate gastrointestinal hormones [38], and can participate in regulating monoamine-based syndrome systems [39]. However, the potential mechanism used by FA to cure depression is unknown. This work used network pharmacological 
methods, detected the active ingredients, targets, and passage, and combined them to obtain the potential mechanism of action of FA on MDD.

UPLC-Q-TOF-MS/MS technology was used to identify FA chemical components. Then, the combination of the literature with the related software resulted in a selection of 12 main active ingredients. Finally, the overlapping target was used to perform the PPI network analysis. DRD2, DRD4, OPRM1, and mTOR were the most relevant targets for the treatment of MDD by FA. DRD2 is divided into two subtypes, D2S and D2L [40], it is a target of many antipsychotic drugs, and its down- or upregulation is closely associate with MDD [41]. mTOR is a receptor serine/ threonine kinase, and in its activated, phosphorylated form can regulate the nerves and promote topical protein synthesis, thereby further promoting the synaptic formation $[42,43]$. A study found that hesperidin exerted an antidepressant effect by activating the hippocampus mTOR pathway [44]. Interestingly, neohesperidin, naringin, and hesperidin were the key components of FA involved in the treatment of MDD, as shown by the hot map. For example, DRD2 and mTOR had a good molecular binding ability than forneohesperidin and hesperidin. Therefore, our hypothesis was that the above ingredients and targets might represent the key elements on the role of FA as antidepressant.

Metascap was used to perform the pathway analysis on 20 core targets. The results showed that FA mainly activates and regulates PI3K-Akt signaling pathway (hsa04151). EGFR tyrosine kinase inhibitor resistance (hsa04080) and ErbB signaling pathway (hsa04072) exert pharmacological effects. The feature to pay attention to is PI3K-Akt signaling pathway, Akt is upstream of the mTOR signaling pathway, and the phosphorylated form can activate the mTOR signaling pathway. Key targets for PI3K/Akt signaling pathway influence the glutamic acid system function, hippocampal neuron apoptosis, and mitochondrial function involved in MDD. Many studies show the main role of TCM as an antidepressant by acting on the PI3K/Akt/mTOR signaling pathway. Caihujialong Tang increases the expression and activity of PI3K in the rat hippocampus to protect neurons, thus exerting an antidepressant effect [45]. Jiaotai Wan regulates $\mathrm{PI} 3 \mathrm{~K} / \mathrm{AKT} / \mathrm{mTOR}$ signaling pathway related proteins to improve depression and reverse behavioral changes in rats [46]. The above evidence generated the hypothesis that FA is effective by acting on AKT, PI3K, and mTOR protein expression.

FA in China is often used as a hydraulic medicine, commonly used to cure gastrointestinal diseases, but the literature does not give any explanation regarding its therapeutic effects on MDD, such as the mitigation of diarrhea caused by MDD. Therefore, our results by UPLC-QTOF-MS/MS technology and system pharmacology revealed a potential mechanism of $\mathrm{FA}$ to treat $\mathrm{MDD}$, providing a reference for the development and use of antidepressants.

\section{Data Availability}

The data used to support the findings of this study are included within the article.

\section{Disclosure}

The funder has no role/influence in this study.

\section{Conflicts of Interest}

The authors declare that they have no conflicts of interest.

\section{Authors' Contributions}

Yating Xie and Ying Liu have contributed equally to this work and share first authorship. XYT integrated the data and wrote the manuscript. ZJL completed the ingredient identification. YXW and ZT accomplished the pharmacological study. ZP and LY executed the literature search. LMM directed the data processing. HM and WQ implemented corrections in the manuscript.

\section{Acknowledgments}

This work was supported by the National Natural Science Foundation of China (nos. 82060724 and 81560651), Science and Technology Project of Jiangxi Health Committee (20210015), and Doctoral Research Startup Fund (no. 2018WBZR009).

\section{References}

[1] F. Aricioğlu, C. Yalcinkaya, C. S. Ozkartal et al., "NLRP1mediated antidepressant effect of ketamine in chronic unpredictable mild stress model in rats," Psychiatry investigation, vol. 17, p. 283, 2020.

[2] M. Li, X. Fu, W. Xie et al., "Effect of early life stress on the epigenetic profiles in depression," Frontiers in Cell and Developmental Biology, vol. 8, p. 867, 2020.

[3] X. Zhou, L. Liu, X. Lan et al., "Polyunsaturated fatty acids metabolism, purine metabolism and inosine as potential independent diagnostic biomarkers for major depressive disorder in children and adolescents," Molecular Psychiatry, vol. 24, no. 10, pp. 1478-1488, 2019.

[4] J. Sawamura, S. Morishita, and J. Ishigooka, "Symmetrical treatment of diagnostic and statistical manual of mental disorders, fifth edition, for major depressive disorders," Source Code for Biology and Medicine, vol. 11, no. 1, pp. 1-14, 2016.

[5] D. Z. Li, "Literature study on the distribution characteristics of TCM syndromes and syndrome elements of depression," Guangming Chinese Medicine, vol. 33, pp. 2781-2783, 2018.

[6] World Health Organization (WHO), Depression and Other Common Mental Disorders: Global Health Estimates, World Health Organization, Geneva, Switzerland, 2017.

[7] S. P. Varghese, O. D. Florentin, and M. M. Koola, "Role of spirituality in the management of major depression and stress-related disorders," Chronic Stress, vol. 5, 2021.

[8] J. Mu, S. Zhang, F. Tang et al., "Mechanism underlying effect of Chaihu Shugan San on major depressive disorder: a network pharmacology-based study," Journal of Traditional Chinese Medicine, vol. 41, no. 2, pp. 338-348, 2021.

[9] Z. Liu, J. Z. Zhang, and W. Y. Gao, "Antinociceptive activity and chemical composition of Wei-Chang-An-Wan extracts," Pharmaceutical Biology, vol. 51, no. 6, pp. 790-797, 2013.

[10] L. Wu, T. Zhang, K. L. Chen et al., "Rapid antidepressant-like effect of Fructus Aurantii depends on cAMP-response 
element binding protein/Brain-derived neurotrophic facto by mediating synaptic transmission," Phytotherapy Research, vol. 35, 2020.

[11] J.-H. Kim, H.-S. Chung, M. Kang et al., "Anti-diabetic effect of standardized herbal formula PM021 consisting of mori folium and aurantii fructus on type II diabetic otsuka long-evans tokushima fatty (OLETF) rats," Diabetes Research and Clinical Practice, vol. 93, no. 2, pp. 198-204, 2011.

[12] D.-Y. Zhou, Q. Xu, X.-Y. Xue, F.-F. Zhang, Y. Jing, and X.-M. Liang, "Rapid qualitative and quantitative analyses of flavanone aglycones inFructus aurantii by HPLC ion-trap MS," Journal of Separation Science, vol. 30, no. 6, pp. 858-867, 2007.

[13] W. Huang, X. Huang, Z. Xing et al., "Meranzin hydrate induces similar effect to fructus aurantii on intestinal motility through activation of $\mathrm{H} 1$ histamine receptors," Journal of Gastrointestinal Surgery, vol. 15, no. 1, pp. 87-96, 2011.

[14] Y. Xu, W. Feng, and J. Y. Guo, "Discussion on antidepressant and its mechanism of fructus aurantii extract," China Clinical Pharmacology and Treatment, vol. 18, pp. 1086-1092, 2013.

[15] X. W. Ye, Y. L. Deng, L. T. Xia, H. M Ren, and J. L Zhang, "Uncovering the mechanism of the effects of Paeoniae Radix Alba on iron-deficiency anaemia through a network pharmacology-based strategy," BMC complementary medicine and therapies, vol. 20, pp. 130-216, 2020.

[16] Y. Deng, H. Ren, X. Ye et al., "Integrated phytochemical analysis based on UPLC-Q-TOF-MS/MS, network pharmacology, and experiment verification to explore the potential mechanism of platycodon grandiflorum for chronic bronchitis," Frontiers in Pharmacology, vol. 11, Article ID 564131, 2020.

[17] Y. L. Deng, X. W. Ye, Y. F. Chen et al., "Chemical characteristics of platycodon grandiflorum and its mechanism in lung cancer treatment," Frontiers in Pharmacology, vol. 11, p. 2316, 2021.

[18] National Pharmacopoeia Commission, Pharmacopoeia of the People's Republic of China. Part I, pp. 246-247, China Medical Science and Technology Press, Beijing, China, 2015.

[19] J. Wang, D. C. Dexter, and Z. A. Shi, "WEB-based GEne SeT AnaLysis toolkit (WebGestalt): update 2013,” Nucleic Acids Research, vol. 41, no. W1, pp. W77-W83, 2013.

[20] B. Gong, Q. Li, and X. H. Hu, "Research progress in chemical composition and pharmacological effects," Southern Forestry Science, vol. 47, pp. 40-45, 2019.

[21] F. T. Wei, The Composition of the Shell and the Study of MultiComponent Pharmacokinetics, Jiangxi University of Traditional Chinese Medicine, Nanchang, China, 2020.

[22] S. S. Xu, W. Xu, and X. M. Zhang, "Prepose of the research progress and quality markers of Chinese medicine tangerine, truth and shell," Chinese herbal medicine, vol. 49, pp. 35-44, 2018.

[23] C. X. Li, Y. H. Yang, and D. S. Leng, "Research progress of chemical composition and pharmacological effects in Fructus Aurantii," Journal of Liaoning University of Traditional Chinese Medicine, vol. 21, pp. 158-161, 2019.

[24] D. L. Liu, G. Z. Deng, and M. H. Yin, "Changes in fingerprints and main flavonoids before and after the shell," Chinese Journal of Experimental Society, vol. 26, pp. 113-118, 2020.

[25] N. J. Abbott, A. A. K. Patabendige, D. E. M. Dolman, S. R. Yusof, and D. J. Begley, "Structure and function of the blood-brain barrier," Neurobiology of Disease, vol. 37, no. 1, pp. 13-25, 2010.

[26] Z. Chang, Z. Y. Han, and Y. S. Han, "Study on the mechanism of anti-depression effect of Radix Bupleuri-Radix Paeoniae
Alba drug pair based on network pharmacology," Journal of Traditional Chinese Medicine, vol. 61, pp. 700-705, 2020.

[27] Y. Ou, M. Luo, Y.-X. Dong et al., "Permeability of novel $4^{\prime}-\mathrm{N}$ substituted (aminomethyl) benzoate-7-substituted nicotinic acid ester derivatives of scutellarein in Caco- 2 cells and in an in vitro model of the blood-brain barrier," Medicinal Chemistry Research, vol. 25, no. 10, pp. 2205-2213, 2016.

[28] M. N. Wang, Q. Y. Mei, and B. Xu, "Scutellarein alleviates dysfunction of blood-brain-barrier initiated by hyperglycemia-stimulated microglia cells," Chinese Pharmacological Bulletin, vol. 36, pp. 1542-1547, 2020.

[29] Y. D. Liang, L. Xie, K. Liu et al., "Bergapten: a review of its pharmacology, pharmacokinetics, and toxicity," Phytotherapy Research, 2021.

[30] Z. Xiao, Nobiletin and Ferulic Acid through Anti-inflammatory and Oxidative Stress and Ghrelin Exerts Antidepressant and Prokinrtic Effects Similar to Zhiqiaodanggui, Xiamen University, Xiamen, China, 2017.

[31] W. Zhao, C. L. Liu, and C. Li, "Pomeli perennose play antidepression by activating mouse Haihua brain area MTOR signaling pathway," Journal of Neuronics, vol. 37, pp. 41-47, 2021.

[32] L. Cai, R. Li, and Q. Q. Wu, "Effects of orange peleton on behavioral and HPA axis of chronic shouldered suppression model," Chinese Journal of Traditional Chinese Medicine, vol. 38, pp. 229-233, 2013.

[33] Y. L. Deng, X. W. Ye, M. M. Liu et al., "Study on mechanism of combination of Platycodonis Radix and Lilii Bulbus with homology of medicine and food in treating pneumonia," Chinese journal of traditional Chinese medicine, vol. 46, pp. 2403-2412, 2021.

[34] H. Y. Ou, X. P. Ye, and J. H. Liu, "Study on the mechanism of peptic colitis in the treatment of ulcerative colitis based on network pharmacology," The Chinese journal of traditional Chinese medicine, vol. 5, pp. 2438-2443, 2019.

[35] N. Wang, NRG1-ErbB4 Signaling Pathways in the Role of Ketamine Antidepressant, Nanjing University, Nanjing, China, 2014.

[36] Y. Xu, W. Feng, and J. Y. Guo, "Discussion on antidepressant and its mechanism of Fructus Aurantii extract," China Clinical Pharmacology and Treatment, vol. 18, pp. 1086-1092, 2013.

[37] M. Wu, H. Zhang, C. Zhou, H. Jia, Z. Ma, and Z. Zou, "Identification of the chemical constituents in aqueous extract of zhi-qiao and evaluation of its antidepressant effect," Molecules, vol. 20, no. 4, pp. 6925-6940, 2015.

[38] X. N. Zhang and S. J. Huang, "Anti-depression in the mood, the shell," Global Chinese Medicine, vol. 7, pp. 77-80, 2014.

[39] Y.-j. Zhang, W. Huang, X. Huang et al., "Fructus Aurantii induced antidepressant effect via its monoaminergic mechanism and prokinetic action in rat," Phytomedicine, vol. 19, no. 12, pp. 1101-1107, 2012.

[40] J. H. Eubanks, M. Djabali, L. Selleri et al., "Structure and linkage of the D2 dopamine receptor and neural cell adhesion molecule genes on human chromosome 11q23," Genomics, vol. 14, no. 4, pp. 1010-1018, 1992.

[41] H. A. Garriock, P. Delgado, M. A. Kling et al., "Number of risk genotypes is a risk factor for major depressive disorder: a case control study," Behavioral and Brain Functions: BBF, vol. 2, pp. 24-28, 2006.

[42] N. Li, B. Lee, R.-J. Liu et al., "mTOR-dependent synapse formation underlies the rapid antidepressant effects of NMDA antagonists," Science, vol. 329, no. 5994, pp. 959-964, 2010. 
[43] C. A. Hoeffer and E. Klann, "mTOR signaling: at the crossroads of plasticity, memory and disease," Trends in Neurosciences, vol. 33, no. 2, pp. 67-75, 2010.

[44] D. Zhao, C. L. Liu, and J. Lee, "Naringin plays an antidepressant role by activating mTOR signaling pathway in hippocampus of mice," Journal of Neuroanatomy, vol. 37, pp. 41-47, 2021.

[45] L. Z. Shang, M. D. Mao, and E. P. Xu, "Pathway of PISK/Akt/ GSKS $\beta / \beta$-catenin in hippocampus issue of rats with depression," Chinese Journal of Experimental Prescription, vol. 26, pp. 12-19, 2020.

[46] F. Man, Study on the Antidepressant Effect Mechanism of JiaotaiPill and its Separated Recipes Based on PI3K/Akt/mTOR Signaling Pathway, Tianjin University of Chinese Medicine, Tianjin, China, 2020. 